\title{
Supplementary information: Impact of Surface Charge Directionality on Membrane Potential in Multi-Ionic Systems
}

\author{
Patricio Ramirez ${ }^{\dagger, *}$, Javier Cervera ${ }^{\ddagger}$, Mubarak Ali $^{\star,} \|$, \\ Saima Nasir ${ }^{\star}, \|$, Wolfgang Ensinger $\|$, and Salvador Mafe ${ }^{\ddagger}$
}

\footnotetext{
${ }^{\dagger}$ Departamento de Física Aplicada, Universitat Politécnica de Valencia, E-46022 Valencia (Spain)

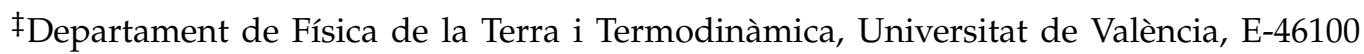
Burjassot (Spain)

^Materials Research Department, GSI Helmholtzzentrum für Schwerionenforschung, D64291 Darmstadt (Germany)

" Department of Material- and Geo-Sciences, Technische Universität Darmstadt, D-64287 Darmstadt (Germany)
}

\section{Contents}

1 General scheme $\quad$ S2

2 Sodium, potassium and chloride ions $\quad$ S3

2.1 Solution I Nanopore interfaces . . . . . . . . . . . S3

2.2 Diffusion potential within the nanopore . . . . . . . . S4

2.3 Liquid junction potentials . . . . . . . . . . . . S5

2.4 Cell membrane potential . . . . . . . . . . . . S6

3 Sodium, potassium and sulfate ions $\quad$ S6

3.1 Solution I Nanopore interfaces . . . . . . . . . . . . . S6

3.2 Diffusion potential within the nanopore . . . . . . . . . S7

3.3 Liquid junction potentials .................. S9 
3.4 Cell membrane potential . . . . . . . . . . . . . . S S10

4 Potassium, chloride and sulfate ions $\quad \mathrm{S10}$

4.1 Solution I Nanopore interfaces . . . . . . . . . . . . . . S10

4.2 Diffusion potential within the nanopore . . . . . . . . . S12

4.3 Liquid junction potentials . . . . . . . . . . . . . . S13

4.4 Cell membrane potential . . . . . . . . . . . . . . . S14

5 Calcium, potassium and chloride ions $\quad \mathrm{S14}$

5.1 Solution I Nanopore interfaces . . . . . . . . . . . . . . S14

5.2 Diffusion potential within the nanopore . . . . . . . . . S16

5.3 Liquid junction potentials . . . . . . . . . . . . . S17

5.4 Cell membrane potential . . . . . . . . . . . . . . S18

$\begin{array}{ll}\text { A Goldmann approximation } & \text { S18 }\end{array}$

$\begin{array}{ll}\text { B The Henderson equation } & \text { S20 }\end{array}$

\section{General scheme}

The schematic view of our system is

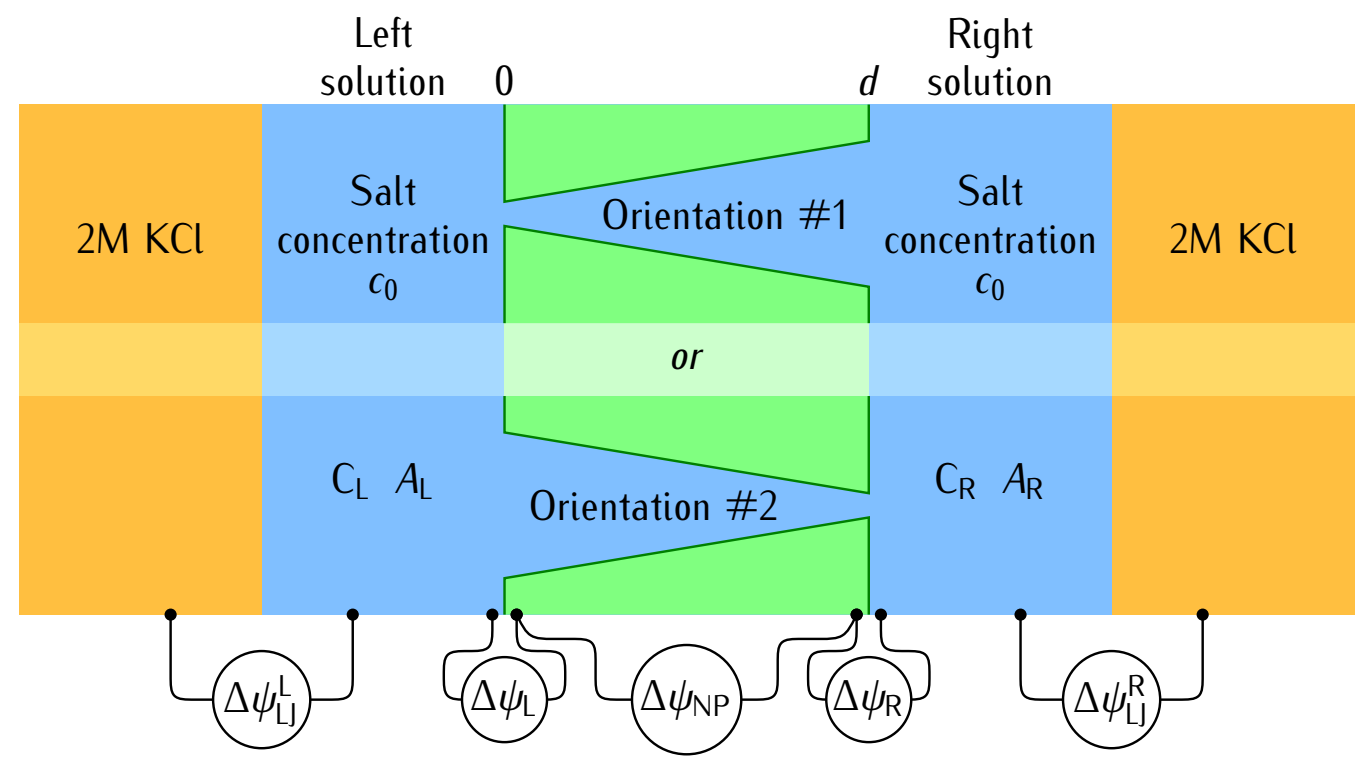

where $\Delta \psi_{\mathrm{L}}=\psi_{\mathrm{L}}-\psi(0), \Delta \psi_{\mathrm{NP}}=\psi(0)-\psi(d)$ and $\Delta \psi_{\mathrm{R}}=\psi(d)-\psi_{\mathrm{R}}$ are the electric potential diference at the left solution I nanopore interface, the 
diffusion potential within the nanopore, and the right solution I nanopore interface, respectively. $\Delta \psi_{\mathrm{LJ}}^{\mathrm{L}}$ and $\Delta \psi_{\mathrm{LJ}}^{\mathrm{R}}$ stand for the liquid junction potential at the electrode salt bridges. All the electric potentials are in $R T / F$ units, where $R$ is the gas constant, $T$ is the temperature, and $F$ is the Faraday constant. $\mathrm{C}_{\mathrm{L}}=\mathrm{Na}^{+}, \mathrm{Ca}^{2+}$, or $\mathrm{K}^{+}$while $\mathrm{C}_{\mathrm{R}}=\mathrm{K}^{+}$always. $\mathrm{A}_{\mathrm{L}}$ and $\mathrm{A}_{\mathrm{R}}=\mathrm{Cl}^{-}$or $\mathrm{SO}_{4}^{2-}$.

\section{Sodium, potassium and chloride ions}

\subsection{Solution I Nanopore interfaces}

We apply first the Donnan equilibria in the left solution I nanopore interface. We consider that we only have $\mathrm{Na}^{+}$(1) and $\mathrm{Cl}^{-}$(3) ions. We apply first the distribution equilibria

$$
\begin{aligned}
& \ln c_{0}+\psi_{\mathrm{L}}=\ln c_{1}(0)+\psi(0) \\
& \ln c_{0}-\psi_{\mathrm{L}}=\ln c_{3}(0)-\psi(0)
\end{aligned}
$$

We solve for $c_{1}(0)$ and $c_{3}(0)$ in both equations

$$
\begin{aligned}
& \ln c_{1}(0)=\ln c_{0}+\overbrace{\psi_{\mathrm{L}}-\psi(0)}^{\Delta \psi_{\mathrm{L}}} \rightarrow c_{1}(0)=c_{0} \mathrm{e}^{\Delta \psi_{\mathrm{L}}} \\
& \ln c_{3}(0)=\ln c_{0}-\left[\psi_{\mathrm{L}}-\psi(0)\right] \rightarrow c_{3}(0)=c_{0} \mathrm{e}^{-\Delta \psi_{\mathrm{L}}}
\end{aligned}
$$

The electroneutrality conditions implies then that

$$
c_{1}(0)=c_{3}(0)+X(0) \rightarrow c_{0} \mathrm{e}^{\Delta \psi_{\mathrm{L}}}=c_{0} \mathrm{e}^{-\Delta \psi_{\mathrm{L}}}+X(0)
$$

or

$$
\mathrm{e}^{2 \Delta \psi_{\mathrm{L}}}-\frac{X(0)}{c_{0}} \mathrm{e}^{\Delta \psi_{\mathrm{L}}}-1=0 \rightarrow u_{\mathrm{L}}^{2}-\frac{X(0)}{c_{0}} u_{\mathrm{L}}-1=0, u_{\mathrm{L}} \equiv \mathrm{e}^{\Delta \psi_{\mathrm{L}}}
$$

whose solution is

$$
u_{\mathrm{L}}=\frac{X(0)}{2 c_{0}}+\sqrt{\left[\frac{X(0)}{2 c_{0}}\right]^{2}+1}
$$

Now the nanopore I right solution interface. We consider that only $\mathrm{K}^{+}$ (2) and $\mathrm{Cl}^{-}$(3) ions are present. As above we apply first the equilibrium equilibria

$$
\begin{aligned}
& \ln c_{2}(d)+\psi(d)=\ln c_{0}+\psi_{\mathrm{R}} \\
& \ln c_{3}(d)-\psi(d)=\ln c_{0}-\psi_{\mathrm{R}}
\end{aligned}
$$


We solve for $c_{2}(d)$ and $c_{3}(d)$

$$
\begin{aligned}
& \ln c_{2}(d)=\ln c_{0}-[\overbrace{\psi(d)-\psi_{\mathrm{R}}}^{\Delta \psi_{\mathrm{R}}}] \rightarrow c_{2}(d)=c_{0} \mathrm{e}^{-\Delta \psi_{\mathrm{R}}} \\
& \ln c_{3}(d)=\ln c_{0}+\left[\psi(d)-\psi_{\mathrm{R}}\right] \rightarrow c_{3}(d)=c_{0} \mathrm{e}^{\Delta \psi_{\mathrm{R}}}
\end{aligned}
$$

We apply the electroneutrality condition

$$
c_{2}(d)=c_{3}(d)+X(d) \rightarrow c_{0} \mathrm{e}^{-\Delta \psi_{\mathrm{R}}}=c_{0} \mathrm{e}^{\Delta \psi_{\mathrm{R}}}+X(d)
$$

that can be written as

$$
\mathrm{e}^{2 \Delta \psi_{\mathrm{R}}}+\frac{X(d)}{c_{0}} \mathrm{e}^{\Delta \psi_{\mathrm{R}}}-1=0 \rightarrow u_{\mathrm{R}}^{2}+\frac{X(d)}{c_{0}} u_{\mathrm{R}}-1=0, u_{\mathrm{R}} \equiv \mathrm{e}^{\Delta \psi_{\mathrm{R}}}
$$

whose solution is

$$
u_{\mathrm{R}}=-\frac{X(d)}{2 c_{0}}+\sqrt{\left[\frac{X(d)}{2 c_{0}}\right]^{2}+1}
$$

\subsection{Diffusion potential within the nanopore}

The conditions for the membrane potential are (i) steady-state and (ii) the total current is zero. The latter condition can be written as

$$
I=0=\sum_{i} z_{i} J_{i}=J_{1}+J_{2}-J_{3}
$$

Using the flux density given by the Goldmann approximation

$$
J_{i}=z_{i} D_{i} \frac{\Delta \psi_{\mathrm{NP}}}{d} \frac{c_{i}(0) \mathrm{e}^{z_{i} \Delta \psi_{\mathrm{NP}}}-c_{i}(d)}{\mathrm{e}^{z_{i} \Delta \psi_{\mathrm{NP}}}-1}
$$

we have

$$
\begin{aligned}
& 0=D_{1} \frac{\Delta \psi_{\mathrm{NP}}}{d} \frac{c_{1}(0) \mathrm{e}^{\Delta \psi_{\mathrm{NP}}}-c_{1}(d)}{\mathrm{e}^{\Delta \psi_{\mathrm{NP}}-1}}+D_{2} \frac{\Delta \psi_{\mathrm{NP}}}{d} \frac{c_{2}(0) \mathrm{e}^{\Delta \psi_{\mathrm{NP}}}-c_{2}(d)}{\mathrm{e}^{\Delta \psi_{\mathrm{NP}}-1}} \\
& +D_{3} \frac{\Delta \psi_{\mathrm{NP}}}{d} \frac{c_{3}(0) \mathrm{e}^{-\Delta \psi_{\mathrm{NP}}}-c_{3}(d)}{\mathrm{e}^{-\Delta \psi_{\mathrm{NP}}-1}}
\end{aligned}
$$

We cancel the common factor $\Delta \psi_{\mathrm{NP}} / d$ and define $v \equiv \mathrm{e}^{\Delta \psi_{\mathrm{NP}}}$ to obtain

$$
0=D_{1} \frac{c_{1}(0) v-c_{1}(d)}{v-1}+D_{2} \frac{c_{2}(0) v-c_{2}(d)}{v-1}+D_{3} \frac{c_{3}(0) v^{-1}-c_{3}(d)}{v^{-1}-1}
$$


We multiply and divide the third term by $-v$

$$
0=D_{1} \frac{c_{1}(0) v-c_{1}(d)}{v-1}+D_{2} \frac{c_{2}(0) v-c_{2}(d)}{v-1}+D_{3} \frac{c_{3}(d) v-c_{3}(0)}{v-1}
$$

which allows to eliminate the denominators. Reordering terms

$$
\begin{aligned}
0= & v\left[D_{1} c_{1}(0)+D_{2} c_{2}(0)+D_{3} c_{3}(d)\right] \\
& -\left[D_{1} c_{1}(d)+D_{2} c_{2}(d)+D_{3} c_{3}(0)\right]
\end{aligned}
$$

or

$$
v=\frac{D_{1} c_{1}(d)+D_{2} c_{2}(d)+D_{3} c_{3}(0)}{D_{1} c_{1}(0)+D_{2} c_{2}(0)+D_{3} c_{3}(d)}
$$

In the previous section we have related the concentrations at $x=0$ and $x=d$ to the external concentrations and Donnan potentials

$$
\begin{array}{rll}
c_{1}(0)=c_{0} u_{\mathrm{L}} & ; & c_{1}(d)=0 \\
c_{2}(0)=0 & ; & c_{2}(d)=c_{0} u_{\mathrm{R}}^{-1} \\
c_{3}(0)=c_{0} u_{\mathrm{L}}^{-1} & ; & c_{3}(d)=c_{0} u_{\mathrm{R}}
\end{array}
$$

so that equation (2.16) can be expressed as

$$
v=\frac{D_{2} u_{\mathrm{R}}^{-1}+D_{3} u_{\mathrm{L}}^{-1}}{D_{1} u_{\mathrm{L}}+D_{3} u_{\mathrm{R}}}
$$

\subsection{Liquid junction potentials}

\section{Left electrode}

The left electrode salt bridge is a $c_{\mathrm{e}}=2 \mathrm{M} \mathrm{KCl}$ solution in agar:

\begin{tabular}{rrcc} 
ion & $z_{i}$ & electrode & left solution \\
\hline $\mathrm{Na}^{+}(1)$ & 1 & 0 & $c_{0}$ \\
$\mathrm{~K}^{+}(2)$ & 1 & $c_{\mathrm{e}}$ & 0 \\
$\mathrm{Cl}^{-}(3)$ & -1 & $c_{\mathrm{e}}$ & $c_{0}$
\end{tabular}

Applying the Henderson equation [equation (B.13) in appendix] the liquid junction potential is estimated as

$$
\Delta \psi_{\mathrm{LJ}}^{\mathrm{L}}=\psi_{\mathrm{LE}}-\psi_{\mathrm{L}}=\frac{\left(D_{1}-D_{3}\right) c_{0}-\left(D_{2}-D_{3}\right) c_{\mathrm{e}}}{\left(D_{1}+D_{3}\right) c_{0}-\left(D_{2}+D_{3}\right) c_{\mathrm{e}}} \ln \left[\frac{\left(D_{1}+D_{3}\right) c_{0}}{\left(D_{2}+D_{3}\right) c_{\mathrm{e}}}\right]
$$




\section{Right electrode}

At the right electrode we also have $c_{\mathrm{e}}=2 \mathrm{M} \mathrm{KCl}$

\begin{tabular}{rrcc} 
ion & $z_{i}$ & right solution & electrode \\
\hline $\mathrm{Na}^{+}(1)$ & 1 & 0 & 0 \\
$\mathrm{~K}^{+}(2)$ & 1 & $c_{0}$ & $c_{\mathrm{e}}$ \\
$\mathrm{Cl}^{-}(3)$ & -1 & $c_{0}$ & $c_{\mathrm{e}}$
\end{tabular}

giving an estimation for the liquid junction potential

$$
\Delta \psi_{\mathrm{LJ}}^{\mathrm{R}}=\psi_{\mathrm{R}}-\psi_{\mathrm{RE}}=\frac{\left(D_{2}-D_{3}\right) c_{\mathrm{e}}-\left(D_{2}-D_{3}\right) c_{0}}{\left(D_{2}+D_{3}\right) c_{\mathrm{e}}-\left(D_{2}+D_{3}\right) c_{0}} \ln \left[\frac{c_{\mathrm{e}}}{c_{0}}\right]
$$

\subsection{Cell membrane potential}

Adding the contributions of the Donnan potentials and the Goldmann potential, the cell membrane potential is

$$
V_{\text {mem }}(\text { corrected })=\frac{R T}{F}\left(\ln u_{\mathrm{L}}+\ln v+\ln u_{\mathrm{R}}\right)
$$

where $u_{\mathrm{L}}, v$ and $u_{\mathrm{R}}$ are given by equations (2.5), (2.18), and (2.10), respectively. From the experimental point of view, equation (2.21) would be the membrane potential corrected for the liquid junction potentials.

If we add the contributions of equations (2.19) and (2.20) we have an estimation of the measured (or uncorrected) experimental values of the membrane potential

$$
V_{\text {mem }}(\text { uncorrected })=\frac{R T}{F}\left(\Delta \psi_{\mathrm{LJ}}^{\mathrm{L}}+\ln u_{\mathrm{L}}+\ln v+\ln u_{\mathrm{R}}+\Delta \psi_{\mathrm{LJ}}^{\mathrm{R}}\right)
$$

\section{Sodium, potassium and sulfate ions}

\subsection{Solution I Nanopore interfaces}

We apply first the Donnan equilibria in the left solution I nanopore interface. We consider that we only have $\mathrm{Na}^{+}(1)$ and $\mathrm{SO}_{4}^{2-}$ (3) ions. We apply first the distribution equilibria

$$
\begin{aligned}
& \ln 2 c_{0}+\psi_{\mathrm{L}}=\ln c_{1}(0)+\psi(0) \\
& \ln c_{0}-2 \psi_{\mathrm{L}}=\ln c_{3}(0)-2 \psi(0)
\end{aligned}
$$


We solve for $c_{1}(0)$ and $c_{3}(0)$ in both equations

$$
\begin{aligned}
& \ln c_{1}(0)=\ln 2 c_{0}+\overbrace{\psi_{\mathrm{L}}-\psi(0)}^{\Delta \psi_{\mathrm{L}}} \rightarrow c_{1}(0)=2 c_{0} \mathrm{e}^{\Delta \psi_{\mathrm{L}}} \\
& \ln c_{3}(0)=\ln c_{0}-2\left[\psi_{\mathrm{L}}-\psi(0)\right] \rightarrow c_{3}(0)=c_{0} \mathrm{e}^{-2 \Delta \psi_{\mathrm{L}}}
\end{aligned}
$$

The electroneutrality conditions implies then that

$$
c_{1}(0)=2 c_{3}(0)+X(0) \rightarrow 2 c_{0} \mathrm{e}^{\Delta \psi_{\mathrm{L}}}=2 c_{0} \mathrm{e}^{-2 \Delta \psi_{\mathrm{L}}}+X(0)
$$

or

$$
\mathrm{e}^{3 \Delta \psi_{\mathrm{L}}}-\frac{X(0)}{2 c_{0}} \mathrm{e}^{2 \Delta \psi_{\mathrm{L}}}-1=0 \rightarrow u_{\mathrm{L}}^{3}-\frac{X(0)}{2 c_{0}} u_{\mathrm{L}}^{2}-1=0, u_{\mathrm{L}} \equiv \mathrm{e}^{\Delta \psi_{\mathrm{L}}}
$$

Now the nanopore I right solution interface. We consider that only $\mathrm{K}^{+}$ (2) and $\mathrm{SO}_{4}^{2-}$ (3) ions are present. As above we apply first the equilibrium equilibria

$$
\begin{aligned}
\ln c_{2}(d)+\psi(d) & =\ln 2 c_{0}+\psi_{\mathrm{R}} \\
\ln c_{3}(d)-2 \psi(d) & =\ln c_{0}-2 \psi_{\mathrm{R}}
\end{aligned}
$$

We solve for $c_{2}(d)$ and $c_{3}(d)$

$$
\begin{aligned}
& \ln c_{2}(d)=\ln 2 c_{0}-[\overbrace{\psi(d)-\psi_{\mathrm{R}}}^{\Delta \psi_{\mathrm{R}}}] \rightarrow c_{2}(d)=2 c_{0} \mathrm{e}^{-\Delta \psi_{\mathrm{R}}} \\
& \ln c_{3}(d)=\ln c_{0}+2\left[\psi(d)-\psi_{\mathrm{R}}\right] \rightarrow c_{3}(d)=c_{0} \mathrm{e}^{2 \Delta \psi_{\mathrm{R}}}
\end{aligned}
$$

We apply the electroneutrality condition

$$
c_{2}(d)=2 c_{3}(d)+X(d) \rightarrow 2 c_{0} \mathrm{e}^{-\Delta \psi_{\mathrm{R}}}=2 c_{0} \mathrm{e}^{2 \Delta \psi_{\mathrm{R}}}+X(d)
$$

that can be written as

$$
\mathrm{e}^{3 \Delta \psi_{\mathrm{R}}}+\frac{X(d)}{2 c_{0}} \mathrm{e}^{\Delta \psi_{\mathrm{R}}}-1=0 \rightarrow u_{\mathrm{R}}^{3}+\frac{X(d)}{2 c_{0}} u_{\mathrm{R}}-1=0, u_{\mathrm{R}} \equiv \mathrm{e}^{\Delta \psi_{\mathrm{R}}}
$$

\subsection{Diffusion potential within the nanopore}

The conditions for the membrane potential are (i) steady-state and (ii) the total current is zero. The latter condition can be written as

$$
I=0=\sum_{i} z_{i} J_{i}=J_{1}+J_{2}-2 J_{3}
$$


Using the flux density given by the Goldmann approximation [equation (A.13)] we have

$$
\begin{aligned}
0= & D_{1} \frac{\Delta \psi_{\mathrm{NP}}}{d} \frac{c_{1}(0) \mathrm{e}^{\Delta \psi_{\mathrm{NP}}}-c_{1}(d)}{\mathrm{e}^{\Delta \psi_{\mathrm{NP}}}-1}+D_{2} \frac{\Delta \psi_{\mathrm{NP}}}{d} \frac{c_{2}(0) \mathrm{e}^{\Delta \psi_{\mathrm{NP}}}-c_{2}(d)}{\mathrm{e}^{\Delta \psi_{\mathrm{NP}}}-1} \\
& +4 D_{3} \frac{\Delta \psi_{\mathrm{NP}}}{d} \frac{c_{3}(0) \mathrm{e}^{-2 \Delta \psi_{\mathrm{NP}}}-c_{3}(d)}{\mathrm{e}^{-2 \Delta \psi_{\mathrm{NP}}}-1}
\end{aligned}
$$

We cancel the common factor $\Delta \psi_{\mathrm{NP}} / d$ and define $v \equiv \mathrm{e}^{\Delta \psi_{\mathrm{NP}}}$ to obtain

$$
0=D_{1} \frac{c_{1}(0) v-c_{1}(d)}{v-1}+D_{2} \frac{c_{2}(0) v-c_{2}(d)}{v-1}+4 D_{3} \frac{c_{3}(0) v^{-2}-c_{3}(d)}{v^{-2}-1}
$$

We multiply and divide the third term by $v^{2}$

$$
0=D_{1} \frac{c_{1}(0) v-c_{1}(d)}{v-1}+D_{2} \frac{c_{2}(0) v-c_{2}(d)}{v-1}+4 D_{3} \frac{c_{3}(0)-c_{3}(d) v^{2}}{1-v^{2}}
$$

This term can also be written as

$$
0=D_{1} \frac{c_{1}(0) v-c_{1}(d)}{v-1}+D_{2} \frac{c_{2}(0) v-c_{2}(d)}{v-1}-4 D_{3} \frac{c_{3}(0)-c_{3}(d) v^{2}}{(v-1)(v+1)}
$$

which allows to eliminate the denominators by multiplying the first and second terms by $v+1$

$$
\begin{aligned}
0= & (v+1) D_{1}\left[c_{1}(0) v-c_{1}(d)\right]+(v+1) D_{2}\left[c_{2}(0) v-c_{2}(d)\right] \\
& -4 D_{3}\left[c_{3}(0)-c_{3}(d) v^{2}\right]
\end{aligned}
$$

We made the multiplication in the first and second terms

$$
\begin{aligned}
0= & D_{1}\left[c_{1}(0) v^{2}+\left[c_{1}(0)-c_{1}(d)\right] v-c_{1}(d)\right] \\
& +D_{2}\left[c_{2}(0) v^{2}+\left[c_{2}(0)-c_{2}(d)\right] v-c_{2}(d)\right] \\
& -4 D_{3}\left[c_{3}(0)-c_{3}(d) v^{2}\right]
\end{aligned}
$$

that can be written as

$$
\begin{aligned}
0= & v^{2}\left[D_{1} c_{1}(0)+D_{2} c_{2}(0)+4 D_{3} c_{3}(d)\right] \\
& +v\left[D_{1}\left[c_{1}(0)-c_{1}(d)\right]+D_{2}\left[c_{2}(0)-c_{2}(d)\right]\right] \\
& -\left[D_{1} c_{1}(d)+D_{2} c_{2}(d)+4 D_{3} c_{3}(0)\right]
\end{aligned}
$$


In the previous section we have related the concentrations at $x=0$ and $x=d$ to the external concentrations and Donnan potentials

$$
\begin{array}{rll}
c_{1}(0)=2 c_{0} u_{\mathrm{L}} & ; & c_{1}(d)=0 \\
c_{2}(0)=0 & ; & c_{2}(d)=2 c_{0} u_{\mathrm{R}}^{-1} \\
c_{3}(0)=c_{0} u_{\mathrm{L}}^{-2} & ; & c_{3}(d)=c_{0} u_{\mathrm{R}}^{2}
\end{array}
$$

so that equation (3.16) can be expressed as

$$
0=v^{2}\left(D_{1} u_{L}+2 D_{3} u_{\mathrm{R}}^{2}\right)+v\left(D_{1} u_{\mathrm{L}}-D_{2} u_{\mathrm{R}}^{-1}\right)-\left(D_{2} u_{\mathrm{R}}^{-1}+2 D_{3} u_{\mathrm{L}}^{-2}\right)
$$

where we have eliminated a factor $2 c_{0}$. The solution of this equation is

$$
\begin{aligned}
v= & -\frac{\left(D_{1} u_{\mathrm{L}}-D_{2} u_{\mathrm{R}}^{-1}\right)}{2\left(D_{1} u_{L}+2 D_{3} u_{\mathrm{R}}^{2}\right)} \\
& +\frac{\sqrt{\left(D_{1} u_{\mathrm{L}}-D_{2} u_{\mathrm{R}}^{-1}\right)^{2}+4\left(D_{1} u_{L}+2 D_{3} u_{\mathrm{R}}^{2}\right)\left(D_{2} u_{\mathrm{R}}^{-1}+2 D_{3} u_{\mathrm{L}}^{-2}\right)}}{2\left(D_{1} u_{L}+2 D_{3} u_{\mathrm{R}}^{2}\right)}
\end{aligned}
$$

\subsection{Liquid junction potentials}

\section{Left electrode}

The left electrode salt bridge is a $c_{\mathrm{e}}=2 \mathrm{M} \mathrm{KCl}$ solution in agar. Giving the index 4 to $\mathrm{Cl}^{-}$ion we have

\begin{tabular}{rrcc} 
ion & $z_{i}$ & electrode & left solution \\
\hline $\mathrm{Na}^{+}(1)$ & 1 & 0 & $2 c_{0}$ \\
$\mathrm{~K}^{+}(2)$ & 1 & $c_{\mathrm{e}}$ & 0 \\
$\mathrm{SO}_{4}^{2-}(3)$ & -2 & 0 & $c_{0}$ \\
$\mathrm{Cl}^{-}(4)$ & -1 & $c_{\mathrm{e}}$ & 0
\end{tabular}

The Henderson equation [equation (B.13)] becomes

$$
\Delta \psi_{\mathrm{LJ}}^{\mathrm{L}}=\psi_{\mathrm{LE}}-\psi_{\mathrm{L}}=\frac{2\left(D_{1}-D_{3}\right) c_{0}-\left(D_{2}-D_{4}\right) c_{\mathrm{e}}}{2\left(D_{1}+2 D_{3}\right) c_{0}-\left(D_{2}+D_{4}\right) c_{\mathrm{e}}} \ln \left(\frac{2 D_{1} c_{0}+4 D_{3} c_{0}}{D_{2} c_{\mathrm{e}}+D_{4} c_{\mathrm{e}}}\right)
$$




\section{Right electrode}

At the right electrode we also have $c_{\mathrm{e}}=2 \mathrm{M} \mathrm{KCl}$

\begin{tabular}{rrcc} 
ion & $z_{i}$ & right solution & electrode \\
\hline $\mathrm{Na}^{+}(1)$ & 1 & 0 & 0 \\
$\mathrm{~K}^{+}(2)$ & 1 & $2 c_{0}$ & $c_{\mathrm{e}}$ \\
$\mathrm{SO}_{4}^{2-}(3)$ & -2 & $c_{0}$ & 0 \\
$\mathrm{Cl}^{-}(4)$ & -1 & 0 & $c_{\mathrm{e}}$
\end{tabular}

giving an estimation for the liquid junction potential

$$
\Delta \psi_{\mathrm{LJ}}^{\mathrm{R}}=\psi_{\mathrm{R}}-\psi_{\mathrm{RE}}=\frac{D_{2}\left(c_{\mathrm{e}}-2 c_{0}\right)+2 D_{3} c_{0}-D_{4} c_{\mathrm{e}}}{D_{2}\left(c_{\mathrm{e}}-2 c_{0}\right)-4 D_{3} c_{0}+D_{4} c_{\mathrm{e}}} \ln \left[\frac{\left(D_{2}+D_{4}\right) c_{\mathrm{e}}}{2\left(D_{2}+2 D_{3}\right) c_{0}}\right]
$$

\subsection{Cell membrane potential}

Adding the contributions of the Donnan potentials and the Goldmann potential, the cell membrane potential is

$$
V_{\text {mem }}(\text { corrected })=\frac{R T}{F}\left(\ln u_{\mathrm{L}}+\ln v+\ln u_{\mathrm{R}}\right)
$$

where $u_{L}, v$ and $u_{R}$ are given by equations (3.4), (3.19), and (3.8), respectively. From the experimental point of view, equation (3.22) would be the membrane potential corrected for the liquid junction potentials.

If we add the contributions of equations (3.20) and (3.21) we have an estimation of the measured (or uncorrected) experimental values of the membrane potential

$$
V_{\text {mem }}(\text { uncorrected })=\frac{R T}{F}\left(\Delta \psi_{\mathrm{LJ}}^{\mathrm{L}}+\ln u_{\mathrm{L}}+\ln v+\ln u_{\mathrm{R}}+\Delta \psi_{\mathrm{LJ}}^{\mathrm{R}}\right)
$$

\section{Potassium, chloride and sulfate ions}

\subsection{Solution I Nanopore interfaces}

We apply first the Donnan equilibria in the left solution I nanopore interface. We consider that we only have $\mathrm{K}^{+}(1)$ and $\mathrm{SO}_{4}^{2-}$ (2) ions. We apply 
first the distribution equilibria

$$
\begin{aligned}
& \ln 2 c_{0}+\psi_{\mathrm{L}}=\ln c_{1}(0)+\psi(0) \\
& \ln c_{0}-2 \psi_{\mathrm{L}}=\ln c_{2}(0)-2 \psi(0)
\end{aligned}
$$

We solve for $c_{1}(0)$ and $c_{2}(0)$ in both equations

$$
\begin{aligned}
& \ln c_{1}(0)=\ln 2 c_{0}+\overbrace{\psi_{\mathrm{L}}-\psi(0)}^{\Delta \psi_{\mathrm{L}}} \rightarrow c_{1}(0)=2 c_{0} \mathrm{e}^{\Delta \psi_{\mathrm{L}}} \\
& \ln c_{2}(0)=\ln c_{0}-2\left[\psi_{\mathrm{L}}-\psi(0)\right] \rightarrow c_{2}(0)=c_{0} \mathrm{e}^{-2 \Delta \psi_{\mathrm{L}}}
\end{aligned}
$$

The electroneutrality conditions implies then that

$$
c_{1}(0)=2 c_{2}(0)+X(0) \rightarrow 2 c_{0} \mathrm{e}^{\Delta \psi_{\mathrm{L}}}=2 c_{0} \mathrm{e}^{-2 \Delta \psi_{\mathrm{L}}}+X(0)
$$

or

$$
\mathrm{e}^{3 \Delta \psi_{\mathrm{L}}}-\frac{X(0)}{2 c_{0}} \mathrm{e}^{2 \Delta \psi_{\mathrm{L}}}-1=0 \rightarrow u_{\mathrm{L}}^{3}-\frac{X(0)}{2 c_{0}} u_{\mathrm{L}}^{2}-1=0, u_{\mathrm{L}} \equiv \mathrm{e}^{\Delta \psi_{\mathrm{L}}}
$$

Now the nanopore I right solution interface. We consider that only $\mathrm{K}^{+}$ (1) and $\mathrm{Cl}^{-}$(3) ions are present. As above we apply first the equilibrium equilibria

$$
\begin{aligned}
& \ln c_{1}(d)+\psi(d)=\ln c_{0}+\psi_{\mathrm{R}} \\
& \ln c_{3}(d)-\psi(d)=\ln c_{0}-\psi_{\mathrm{R}}
\end{aligned}
$$

We solve for $c_{1}(d)$ and $c_{3}(d)$

$$
\begin{aligned}
& \ln c_{1}(d)=\ln c_{0}-[\overbrace{\psi(d)-\psi_{\mathrm{R}}}^{\Delta \psi_{\mathrm{R}}}] \rightarrow c_{1}(d)=c_{0} \mathrm{e}^{-\Delta \psi_{\mathrm{R}}} \\
& \ln c_{3}(d)=\ln c_{0}+\left[\psi(d)-\psi_{\mathrm{R}}\right] \rightarrow c_{3}(d)=c_{0} \mathrm{e}^{\Delta \psi_{\mathrm{R}}}
\end{aligned}
$$

We apply the electroneutrality condition

$$
c_{1}(d)=c_{3}(d)+X(d) \rightarrow c_{0} \mathrm{e}^{-\Delta \psi_{\mathrm{R}}}=c_{0} \mathrm{e}^{\Delta \psi_{\mathrm{R}}}+X(d)
$$

that can be written as

$$
\mathrm{e}^{2 \Delta \psi_{\mathrm{R}}}+\frac{X(d)}{c_{0}} \mathrm{e}^{\Delta \psi_{\mathrm{R}}}-1=0 \rightarrow u_{\mathrm{R}}^{2}+\frac{X(d)}{c_{0}} u_{\mathrm{R}}-1=0, u_{\mathrm{R}} \equiv \mathrm{e}^{\Delta \psi_{\mathrm{R}}}
$$

the solution of which is

$$
u_{\mathrm{R}}=-\frac{X(d)}{2 c_{0}}+\sqrt{\left[\frac{X(d)}{2 c_{0}}\right]^{2}+1}
$$




\subsection{Diffusion potential within the nanopore}

The conditions for the membrane potential are (i) steady-state and (ii) the total current is zero. The latter condition can be written as

$$
I=0=\sum_{i} z_{i} J_{i}=J_{1}-2 J_{2}-J_{3}
$$

Using the flux density given by the Goldmann approximation [equation (A.13)] we have

$$
\begin{aligned}
0= & D_{1} \frac{\Delta \psi_{\mathrm{NP}}}{d} \frac{c_{1}(0) \mathrm{e}^{\Delta \psi_{\mathrm{NP}}}-c_{1}(d)}{\mathrm{e}^{\Delta \psi_{\mathrm{NP}}}-1}+4 D_{2} \frac{\Delta \psi_{\mathrm{NP}}}{d} \frac{c_{2}(0) \mathrm{e}^{-2 \Delta \psi_{\mathrm{NP}}}-c_{2}(d)}{\mathrm{e}^{-2 \Delta \psi_{\mathrm{NP}}-1}} \\
& +D_{3} \frac{\Delta \psi_{\mathrm{NP}}}{d} \frac{c_{3}(0) \mathrm{e}^{-\Delta \psi_{\mathrm{NP}}}-c_{3}(d)}{\mathrm{e}^{-\Delta \psi_{\mathrm{NP}}}-1}
\end{aligned}
$$

We cancel the common factor $\Delta \psi_{\mathrm{NP}} / d$ and define $v \equiv \mathrm{e}^{\Delta \psi_{\mathrm{NP}}}$ to obtain

$$
0=D_{1} \frac{c_{1}(0) v-c_{1}(d)}{v-1}+4 D_{2} \frac{c_{2}(0) v^{-2}-c_{2}(d)}{v^{-2}-1}+D_{3} \frac{c_{3}(0) v^{-1}-c_{3}(d)}{v^{-1}-1}
$$

We multiply and divide the second term by $v^{2}$ and the third term by $v$

$$
0=D_{1} \frac{c_{1}(0) v-c_{1}(d)}{v-1}+4 D_{2} \frac{c_{2}(0)-c_{2}(d) v^{2}}{1-v^{2}}+D_{3} \frac{c_{3}(0)-c_{3}(d) v}{1-v}
$$

Because $1-v^{2}=(1-v)(1+v)$ we can eliminate the denominators by mutiplying the first and third terms by $v+1$

$$
\begin{aligned}
0= & (v+1) D_{1}\left[c_{1}(0) v-c_{1}(d)\right]-4 D_{2}\left[c_{2}(0)-c_{2}(d) v^{2}\right] \\
& -(v+1) D_{3}\left[c_{3}(0)-c_{3}(d) v\right]
\end{aligned}
$$

We made the multiplication in the first and third terms

$$
\begin{aligned}
0= & D_{1}\left[c_{1}(0) v^{2}+\left[c_{1}(0)-c_{1}(d)\right] v-c_{1}(d)\right] \\
& -4 D_{2}\left[c_{2}(0)-c_{2}(d) v^{2}\right] \\
& -D_{3}\left[-c_{3}(d) v^{2}+\left[c_{3}(0)-c_{3}(d)\right] v+c_{3}(0)\right]
\end{aligned}
$$

that can be written as

$$
\begin{aligned}
0= & v^{2}\left[D_{1} c_{1}(0)+4 D_{2} c_{2}(d)+D_{3} c_{3}(d)\right] \\
& +v\left[D_{1}\left[c_{1}(0)-c_{1}(d)\right]+D_{3}\left[c_{3}(d)-c_{3}(0)\right]\right] \\
& -\left[D_{1} c_{1}(d)+4 D_{2} c_{2}(0)+D_{3} c_{3}(0)\right]
\end{aligned}
$$


The relation of the concentrations at $x=0$ and $x=d$ to the external concentrations and Donnan potentials is

$$
\begin{array}{ll}
c_{1}(0)=2 c_{0} u_{\mathrm{L}} ; & c_{1}(d)=c_{0} u_{\mathrm{R}}^{-1} \\
c_{2}(0)=c_{0} u_{\mathrm{L}}^{-2} & ; \quad c_{2}(d)=0 \\
c_{3}(0)=0 \quad & c_{3}(d)=c_{0} u_{\mathrm{R}}
\end{array}
$$

so that equation (4.16) can be expressed as

$0=v^{2}\left(2 D_{1} u_{L}+D_{3} u_{\mathrm{R}}\right)+v\left[D_{1}\left(2 u_{\mathrm{L}}-u_{\mathrm{R}}^{-1}\right)+D_{3} u_{\mathrm{R}}\right]-\left(D_{1} u_{\mathrm{R}}^{-1}+4 D_{2} u_{\mathrm{L}}^{-2}\right)$

where we have eliminated a factor $c_{0}$. The solution of this equation is

$$
\begin{aligned}
v= & -\frac{\left[D_{1}\left(2 u_{\mathrm{L}}-u_{\mathrm{R}}^{-1}\right)+D_{3} u_{\mathrm{R}}\right]}{2\left(2 D_{1} u_{L}+D_{3} u_{\mathrm{R}}\right)} \\
& +\frac{\sqrt{\left[D_{1}\left(2 u_{\mathrm{L}}-u_{\mathrm{R}}^{-1}\right)+D_{3} u_{\mathrm{R}}\right]^{2}+4\left(2 D_{1} u_{L}+D_{3} u_{\mathrm{R}}\right)\left(D_{1} u_{\mathrm{R}}^{-1}+4 D_{2} u_{\mathrm{L}}^{-2}\right)}}{2\left(2 D_{1} u_{L}+D_{3} u_{\mathrm{R}}\right)}
\end{aligned}
$$

\subsection{Liquid junction potentials}

\section{Left electrode}

The left electrode salt bridge is a $c_{\mathrm{e}}=2 \mathrm{M} \mathrm{KCl}$ solution in agar.

\begin{tabular}{rrcc} 
ion & $z_{i}$ & electrode & left solution \\
\hline $\mathrm{K}^{+}(1)$ & 1 & $c_{\mathrm{e}}$ & $2 c_{0}$ \\
$\mathrm{SO}_{4}^{2-}(2)$ & -2 & 0 & $c_{0}$ \\
$\mathrm{Cl}^{-}(3)$ & -1 & $c_{\mathrm{e}}$ & 0
\end{tabular}

The Henderson equation [equation (B.13)] becomes

$$
\Delta \psi_{\mathrm{LJ}}^{\mathrm{L}}=\psi_{\mathrm{LE}}-\psi_{\mathrm{L}}=\frac{2\left(D_{1}-D_{2}\right) c_{0}-\left(D_{1}-D_{3}\right) c_{\mathrm{e}}}{2\left(D_{1}+2 D_{2}\right) c_{0}-\left(D_{1}+D_{3}\right) c_{\mathrm{e}}} \ln \left(\frac{2 D_{1} c_{0}+4 D_{2} c_{0}}{D_{1} c_{\mathrm{e}}+D_{3} c_{\mathrm{e}}}\right)
$$

\section{Right electrode}

At the right electrode we also have $c_{\mathrm{e}}=2 \mathrm{M} \mathrm{KCl}$ 


\begin{tabular}{rrcc} 
ion & $z_{i}$ & right solution & electrode \\
\hline $\mathrm{K}^{+}(1)$ & 1 & $c_{0}$ & $c_{\mathrm{e}}$ \\
$\mathrm{SO}_{4}^{2-}(2)$ & -2 & 0 & 0 \\
$\mathrm{Cl}^{-}(3)$ & -1 & $c_{0}$ & $c_{\mathrm{e}}$
\end{tabular}

giving an estimation for the liquid junction potential

$$
\Delta \psi_{\mathrm{LJ}}^{\mathrm{R}}=\psi_{\mathrm{R}}-\psi_{\mathrm{RE}}=\frac{D_{1}\left(c_{\mathrm{e}}-c_{0}\right)-D_{3}\left(c_{\mathrm{e}}-c_{0}\right)}{D_{1}\left(c_{\mathrm{e}}-c_{0}\right)+D_{3}\left(c_{\mathrm{e}}-c_{0}\right)} \ln \left(\frac{c_{\mathrm{e}}}{c_{0}}\right)
$$

\subsection{Cell membrane potential}

Adding the contributions of the Donnan potentials and the Goldmann potential, the cell membrane potential is

$$
V_{\text {mem }}(\text { corrected })=\frac{R T}{F}\left(\ln u_{\mathrm{L}}+\ln v+\ln u_{\mathrm{R}}\right)
$$

where $u_{\mathrm{L}}, v$ and $u_{\mathrm{R}}$ are given by equations (4.4), (4.19), and (4.9), respectively. From the experimental point of view, equation (4.22) would be the membrane potential corrected for the liquid junction potentials.

If we add the contributions of equations (4.20) and (4.21) we have an estimation of the measured (or uncorrected) experimental values of the membrane potential

$$
V_{\text {mem }}(\text { uncorrected })=\frac{R T}{F}\left(\Delta \psi_{\mathrm{LJ}}^{\mathrm{L}}+\ln u_{\mathrm{L}}+\ln v+\ln u_{\mathrm{R}}+\Delta \psi_{\mathrm{LJ}}^{\mathrm{R}}\right)
$$

\section{Calcium, potassium and chloride ions}

\subsection{Solution I Nanopore interfaces}

We apply first the Donnan equilibria in the left solution I nanopore interface. We consider that we only have $\mathrm{Ca}^{2+}$ (1) and $\mathrm{Cl}^{-}$(2) ions. We apply first the distribution equilibria

$$
\begin{aligned}
& \ln c_{0}+2 \psi_{\mathrm{L}}=\ln c_{1}(0)+2 \psi(0) \\
& \ln 2 c_{0}-\psi_{\mathrm{L}}=\ln c_{2}(0)-\psi(0)
\end{aligned}
$$


We solve for $c_{1}(0)$ and $c_{2}(0)$ in both equations

$$
\begin{aligned}
& \ln c_{1}(0)=\ln c_{0}+2[\overbrace{\psi_{\mathrm{L}}-\psi(0)}^{\Delta \psi_{\mathrm{L}}}] \rightarrow c_{1}(0)=c_{0} \mathrm{e}^{2 \Delta \psi_{\mathrm{L}}} \\
& \ln c_{2}(0)=\ln 2 c_{0}-\left[\psi_{\mathrm{L}}-\psi(0)\right] \rightarrow c_{2}(0)=2 c_{0} \mathrm{e}^{-\Delta \psi_{\mathrm{L}}}
\end{aligned}
$$

The electroneutrality conditions implies then that

$$
2 c_{1}(0)=c_{2}(0)+X(0) \rightarrow 2 c_{0} \mathrm{e}^{2 \Delta \psi_{\mathrm{L}}}=2 c_{0} \mathrm{e}^{-\Delta \psi_{\mathrm{L}}}+X(0)
$$

or

$$
\mathrm{e}^{3 \Delta \psi_{\mathrm{L}}}-\frac{X(0)}{2 c_{0}} \mathrm{e}^{\Delta \psi_{\mathrm{L}}}-1=0 \rightarrow u_{\mathrm{L}}^{3}-\frac{X(0)}{2 c_{0}} u_{\mathrm{L}}-1=0, u_{\mathrm{L}} \equiv \mathrm{e}^{\Delta \psi_{\mathrm{L}}}
$$

Now the nanopore I right solution interface. We consider that only $\mathrm{K}^{+}$ (3) and $\mathrm{Cl}^{-}$(2) ions are present. As above we apply first the equilibrium equilibria

$$
\begin{aligned}
& \ln c_{3}(d)+\psi(d)=\ln c_{0}+\psi_{\mathrm{R}} \\
& \ln c_{2}(d)-\psi(d)=\ln c_{0}-\psi_{\mathrm{R}}
\end{aligned}
$$

We solve for $c_{3}(d)$ and $c_{2}(d)$

$$
\begin{aligned}
& \ln c_{3}(d)=\ln c_{0}-[\overbrace{\psi(d)-\psi_{\mathrm{R}}}^{\Delta \psi_{\mathrm{R}}}] \rightarrow c_{3}(d)=c_{0} \mathrm{e}^{-\Delta \psi_{\mathrm{R}}} \\
& \ln c_{2}(d)=\ln c_{0}+\left[\psi(d)-\psi_{\mathrm{R}}\right] \rightarrow c_{2}(d)=c_{0} \mathrm{e}^{\Delta \psi_{\mathrm{R}}}
\end{aligned}
$$

We apply the electroneutrality condition

$$
c_{3}(d)=c_{2}(d)+X(d) \rightarrow c_{0} \mathrm{e}^{-\Delta \psi_{\mathrm{R}}}=c_{0} \mathrm{e}^{\Delta \psi_{\mathrm{R}}}+X(d)
$$

that can be written as

$$
\mathrm{e}^{2 \Delta \psi_{\mathrm{R}}}+\frac{X(d)}{c_{0}} \mathrm{e}^{\Delta \psi_{\mathrm{R}}}-1=0 \rightarrow u_{\mathrm{R}}^{2}+\frac{X(d)}{c_{0}} u_{\mathrm{R}}-1=0, u_{\mathrm{R}} \equiv \mathrm{e}^{\Delta \psi_{\mathrm{R}}}
$$

the solution of which is

$$
u_{\mathrm{R}}=-\frac{X(d)}{2 c_{0}}+\sqrt{\left[\frac{X(d)}{2 c_{0}}\right]^{2}+1}
$$




\subsection{Diffusion potential within the nanopore}

The conditions for the membrane potential are (i) steady-state and (ii) the total current is zero. The latter condition can be written as

$$
I=0=\sum_{i} z_{i} J_{i}=2 J_{1}-J_{2}+J_{3}
$$

Using the flux density given by the Goldmann approximation [equation (A.13)] we have

$$
\begin{aligned}
0= & 4 D_{1} \frac{\Delta \psi_{\mathrm{NP}}}{d} \frac{c_{1}(0) \mathrm{e}^{2 \Delta \psi_{\mathrm{NP}}}-c_{1}(d)}{\mathrm{e}^{2 \Delta \psi_{\mathrm{NP}}}-1}+D_{2} \frac{\Delta \psi_{\mathrm{NP}}}{d} \frac{c_{2}(0) \mathrm{e}^{-\Delta \psi_{\mathrm{NP}}}-c_{2}(d)}{\mathrm{e}^{-\Delta \psi_{\mathrm{NP}}-1}} \\
& +D_{3} \frac{\Delta \psi_{\mathrm{NP}}}{d} \frac{c_{3}(0) \mathrm{e}^{\Delta \psi_{\mathrm{NP}}}-c_{3}(d)}{\mathrm{e}^{\Delta \psi_{\mathrm{NP}}-1}}
\end{aligned}
$$

We cancel the common factor $\Delta \psi_{\mathrm{NP}} / d$ and define $v \equiv \mathrm{e}^{\Delta \psi_{\mathrm{NP}}}$ to obtain

$$
0=4 D_{1} \frac{c_{1}(0) v^{2}-c_{1}(d)}{v^{2}-1}+D_{2} \frac{c_{2}(0) v^{-1}-c_{2}(d)}{v^{-1}-1}+D_{3} \frac{c_{3}(0) v-c_{3}(d)}{v-1}
$$

We multiply and divide the second term by $v$

$$
0=4 D_{1} \frac{c_{1}(0) v^{2}-c_{1}(d)}{v^{2}-1}+D_{2} \frac{c_{2}(0)-c_{2}(d) v}{1-v}+D_{3} \frac{c_{3}(0) v-c_{3}(d)}{v-1}
$$

Because $v^{2}-1=(v-1)(v-1)$ we can eliminate the denominators by mutiplying the second and third terms by $v+1$

$$
\begin{aligned}
0= & 4 D_{1}\left[c_{1}(0) v^{2}-c_{1}(d)\right]-(v+1) D_{2}\left[c_{2}(0)-c_{2}(d) v\right] \\
& +(v+1) D_{3}\left[c_{3}(0) v-c_{3}(d)\right]
\end{aligned}
$$

We made the multiplication in the second and third terms

$$
\begin{aligned}
0= & 4 D_{1}\left[c_{1}(0) v^{2}-c_{1}(d)\right] \\
& -D_{2}\left[-c_{2}(d) v^{2}+\left[c_{2}(0)-c_{2}(d)\right] v+c_{2}(0)\right] \\
& +D_{3}\left[c_{3}(0) v^{2}+\left[c_{3}(0)-c_{3}(d)\right] v-c_{3}(d)\right]
\end{aligned}
$$

that can be written as

$$
\begin{aligned}
0= & v^{2}\left[4 D_{1} c_{1}(0)+D_{2} c_{2}(d)+D_{3} c_{3}(0)\right] \\
& +v\left[-D_{2}\left[c_{2}(0)-c_{2}(d)\right]+D_{3}\left[c_{3}(0)-c_{3}(d)\right]\right] \\
& -\left[4 D_{1} c_{1}(d)+D_{2} c_{2}(0)+D_{3} c_{3}(d)\right]
\end{aligned}
$$


The relation of the concentrations at $x=0$ and $x=d$ to the external concentrations and Donnan potentials is

$$
\begin{array}{lll}
c_{1}(0)=c_{0} u_{\mathrm{L}}^{2} & ; \quad c_{1}(d)=0 \\
c_{2}(0)=2 c_{0} u_{\mathrm{L}}^{-1} & ; \quad c_{2}(d)=c_{0} u_{\mathrm{R}} \\
c_{3}(0)=0 & ; \quad c_{3}(d)=c_{0} u_{\mathrm{R}}^{-1}
\end{array}
$$

so that equation (5.16) can be expressed as

$$
0=v^{2}\left(4 D_{1} u_{L}^{2}+D_{2} u_{\mathrm{R}}\right)+v\left[D_{2}\left(u_{\mathrm{R}}-2 u_{\mathrm{L}}^{-1}\right)-D_{3} u_{\mathrm{R}}^{-1}\right]-\left(2 D_{2} u_{\mathrm{L}}^{-1}+D_{3} u_{\mathrm{R}}^{-1}\right)
$$

where we have eliminated a factor $c_{0}$. The solution of this equation is

$$
\begin{aligned}
v= & -\frac{\left[D_{2}\left(u_{\mathrm{R}}-2 u_{\mathrm{L}}^{-1}\right)-D_{3} u_{\mathrm{R}}^{-1}\right]}{2\left(4 D_{1} u_{L}^{2}+D_{2} u_{\mathrm{R}}\right)} \\
& +\frac{\sqrt{\left[D_{2}\left(u_{\mathrm{R}}-2 u_{\mathrm{L}}^{-1}\right)-D_{3} u_{\mathrm{R}}^{-1}\right]^{2}+4\left(4 D_{1} u_{L}^{2}+D_{2} u_{\mathrm{R}}\right)\left(2 D_{2} u_{\mathrm{L}}^{-1}+D_{3} u_{\mathrm{R}}^{-1}\right)}}{2\left(4 D_{1} u_{L}^{2}+D_{2} u_{\mathrm{R}}\right)}
\end{aligned}
$$

\subsection{Liquid junction potentials}

\section{Left electrode}

The left electrode salt bridge is a $c_{\mathrm{e}}=2 \mathrm{M} \mathrm{KCl}$ solution in agar.

\begin{tabular}{rrcc} 
ion & $z_{i}$ & electrode & left solution \\
\hline $\mathrm{Ca}^{2+}(1)$ & 2 & 0 & $c_{0}$ \\
$\mathrm{Cl}^{-}(2)$ & -1 & $c_{\mathrm{e}}$ & $2 c_{0}$ \\
$\mathrm{~K}^{+}(3)$ & 1 & $c_{\mathrm{e}}$ & 0
\end{tabular}

Using the Henderson equation [equation (B.13), see appendix] we have

$$
\Delta \psi_{\mathrm{LJ}}^{\mathrm{L}}=\psi_{\mathrm{LE}}-\psi_{\mathrm{L}}=\frac{2\left(D_{1}-D_{2}\right) c_{0}+\left(D_{2}-D_{3}\right) c_{\mathrm{e}}}{2\left(2 D_{1}+D_{2}\right) c_{0}-\left(D_{2}+D_{3}\right) c_{\mathrm{e}}} \ln \left(\frac{4 D_{1} c_{0}+2 D_{2} c_{0}}{D_{2} c_{\mathrm{e}}+D_{3} c_{\mathrm{e}}}\right)
$$

\section{Right electrode}

At the right electrode we also have $c_{\mathrm{e}}=2 \mathrm{M} \mathrm{KCl}$ 


\begin{tabular}{rccc} 
ion & $z_{i}$ & right solution & electrode \\
\hline $\mathrm{Ca}^{2+}(1)$ & +2 & 0 & 0 \\
$\mathrm{Cl}^{-}(2)$ & -1 & $c_{0}$ & $c_{\mathrm{e}}$ \\
$\mathrm{K}^{+}(3)$ & +1 & $c_{0}$ & $c_{\mathrm{e}}$
\end{tabular}

giving an estimation for the liquid junction potential

$$
\Delta \psi_{\mathrm{LJ}}^{\mathrm{R}}=\psi_{\mathrm{R}}-\psi_{\mathrm{RE}}=\frac{-D_{2}\left(c_{\mathrm{e}}-c_{0}\right)+D_{3}\left(c_{\mathrm{e}}-c_{0}\right)}{D_{2}\left(c_{\mathrm{e}}-c_{0}\right)+D_{3}\left(c_{\mathrm{e}}-c_{0}\right)} \ln \left(\frac{c_{\mathrm{e}}}{c_{0}}\right)
$$

\subsection{Cell membrane potential}

Adding the contributions of the Donnan potentials and the Goldmann potential, the cell membrane potential is

$$
V_{\text {mem }}(\text { corrected })=\frac{R T}{F}\left(\ln u_{\mathrm{L}}+\ln v+\ln u_{\mathrm{R}}\right)
$$

where $u_{\mathrm{L}}, v$ and $u_{\mathrm{R}}$ are given by equations (5.4), (5.19), and (5.9), respectively. From the experimental point of view, equation (5.22) would be the membrane potential corrected for the liquid junction potentials.

If we add the contributions of equations (5.20) and (5.21) we have an estimation of the measured (or uncorrected) experimental values of the membrane potential

$$
V_{\text {mem }}(\text { uncorrected })=\frac{R T}{F}\left(\Delta \psi_{\mathrm{LJ}}^{\mathrm{L}}+\ln u_{\mathrm{L}}+\ln v+\ln u_{\mathrm{R}}+\Delta \psi_{\mathrm{LJ}}^{\mathrm{R}}\right)
$$

\section{A Goldmann approximation}

Consider the flux density of ion $i$ in 1D

$$
J_{i}=-D_{i} \frac{\mathrm{d} c_{i}}{\mathrm{~d} x}-z_{i} D_{i} c_{i} \frac{\mathrm{d} \psi}{\mathrm{d} x}
$$

The Goldmann aproximation consists of linearizing the electric potential gradient (constant field approximation)

$$
\frac{\mathrm{d} \psi}{\mathrm{d} x} \approx \frac{\psi(d)-\psi(0)}{d}=-\frac{\Delta \psi_{\mathrm{NP}}}{d}
$$


The flux density becomes

$$
J_{i}=-D_{i} \frac{\mathrm{d} c_{i}}{\mathrm{~d} x}+z_{i} D_{i} c_{i} \frac{\Delta \psi_{\mathrm{NP}}}{d}
$$

To calculate $J_{i}$ we assume the steady-state condition

$$
\frac{\mathrm{d} J_{i}}{\mathrm{~d} x}=0 \rightarrow \frac{\mathrm{d}^{2} c_{i}}{\mathrm{~d} x^{2}}-z_{i} \frac{\Delta \psi_{\mathrm{NP}}}{\mathrm{d}} \frac{\mathrm{d} c_{i}}{\mathrm{~d} x}=0
$$

The characteristic polynomial of this differential equation is

$$
\lambda_{i}^{2}-z_{i} \frac{\Delta \psi_{\mathrm{NP}}}{d} \lambda_{i}=0 \rightarrow \lambda_{i}=\left\{\begin{array}{l}
0 \\
z_{i} \Delta \psi_{\mathrm{NP}} / d
\end{array}\right.
$$

so that the solution of equation (A.4) is

$$
c_{i}(x)=A+B \mathrm{e}^{z_{i} \Delta \psi_{\mathrm{NP}} x / d} \text {, with } A, B \text { constants }
$$

To know the values of $A$ and $B$ we apply the boundary conditions

$$
\begin{aligned}
& c_{i}(0)=A+B \\
& c_{i}(d)=A+B \mathrm{e}^{z_{i} \Delta \psi_{\mathrm{NP}}}
\end{aligned}
$$

We can obtain $B$ by substracting the first to the second equation

$$
c_{i}(d)-c_{i}(0)=B\left(\mathrm{e}^{z_{i} \Delta \psi_{\mathrm{NP}}}-1\right) \rightarrow B=\frac{c_{i}(d)-c_{i}(0)}{\mathrm{e}^{z_{i} \Delta \psi_{\mathrm{NP}}-1}}
$$

With $B$ we can obtain $A$

$$
A=c_{i}(0)-B=\frac{c_{i}(0) \mathrm{e}_{z_{i} \Delta \psi}-c_{i}(d)}{\mathrm{e}^{z_{i} \Delta \psi_{\mathrm{NP}}}-1}
$$

The final solution for $c_{i}(x)$ is

$$
c_{i}(x)=\frac{\left.c_{i}(0) \mathrm{e}^{z_{i} \Delta \psi_{\mathrm{NP}}}-c_{i}(d)+\left[c_{i}(d)-c_{i}(0)\right]\right] \mathrm{e}^{z_{i} \Delta \psi_{\mathrm{NP}} x / d}}{\mathrm{e}^{z_{i} \Delta \psi_{\mathrm{NP}}}-1}
$$

Its derivative with $x$ is

$$
\frac{\mathrm{d} c_{i}}{\mathrm{~d} x}=z_{i} \frac{\Delta \psi_{\mathrm{NP}}}{d} \frac{c_{i}(d)-c_{i}(0)}{\mathrm{e}^{z_{i} \Delta \psi_{\mathrm{NP}}-1}} \mathrm{e}^{z_{i} \Delta \psi_{\mathrm{NP}} x / d}
$$

and the density flux becomes

$$
\begin{aligned}
J_{i}= & -D_{i} z_{i} \frac{\Delta \psi_{\mathrm{NP}}}{d} \frac{c_{i}(d)-c_{i}(0)}{\mathrm{e}^{z_{i} \Delta \psi_{\mathrm{NP}}-1} \mathrm{e}^{z_{i} \Delta \psi_{\mathrm{NP}} x / d}} \\
& +z_{i} D_{i} \frac{\Delta \psi_{\mathrm{NP}}}{d} \frac{c_{i}(0) \mathrm{e}^{z_{i} \Delta \psi_{\mathrm{NP}}}-c_{i}(d)}{\mathrm{e}^{z_{i} \Delta \psi_{\mathrm{NP}}}-1}+z_{i} D_{i} \frac{\Delta \psi_{\mathrm{NP}}}{d} \frac{c_{i}(d)-c_{i}(0)}{\mathrm{e}^{z_{i} \Delta \psi_{\mathrm{NP}}-1}} \mathrm{e}^{z_{i} \Delta \psi_{\mathrm{NP}} x / d}
\end{aligned}
$$

that simplifies to

$$
J_{i}=z_{i} D_{i} \frac{\Delta \psi_{\mathrm{NP}}}{d} \frac{c_{i}(0) \mathrm{e}^{z_{i} \Delta \psi_{\mathrm{NP}}}-c_{i}(d)}{\mathrm{e}^{z_{i} \Delta \psi_{\mathrm{NP}}-1}}
$$




\section{B The Henderson equation}

Assume an interface that separates two solutions, named left (L) and right (R). Far from the interface we know the concentration value of a series of ions $i: c_{i \mathrm{~L}}, c_{i \mathrm{R}}$. To calculate the change of the electric potential across the interface, the Henderson equation assumes (i) that the current is null, (ii) that the ionic concentration varies linearly from the left to the right solution (both at bulk), and (iii) that the diffusion coefficients are constant.

We start with the 1D Nernst-Planck equation to calculate the ionic flux of ionic species $i$

$$
J_{i}=-D_{i} \frac{\mathrm{d} c_{i}}{\mathrm{~d} x}-z_{i} D_{i} c_{i} \frac{\mathrm{d} \psi}{\mathrm{d} x}
$$

If we consider that the length between the bulk of the left and right solutions is $l$ and assume a linear variation of the concentration we have

$$
c_{i}(x)=c_{i \mathrm{~L}}+\frac{c_{i \mathrm{R}}-c_{i \mathrm{~L}}}{l} x
$$

Substituting this expression in the Nernst-Planck equation we have

$$
J_{i}=-D_{i} \frac{c_{i \mathrm{R}}-c_{i \mathrm{~L}}}{l}-z_{i} D_{i}\left(c_{i \mathrm{~L}}+\frac{c_{i \mathrm{R}}-c_{i \mathrm{~L}}}{l} x\right) \frac{\mathrm{d} \psi}{\mathrm{d} x}
$$

The condition for zero current reads

$$
0=\sum_{i} z_{i} I_{i}
$$

With equation (B.3) this condition becomes

$$
0=\sum_{i} z_{i} D_{i} \frac{c_{i \mathrm{R}}-c_{i \mathrm{~L}}}{l}+\sum_{i} z_{i}^{2} D_{i}\left(c_{i \mathrm{~L}}+\frac{c_{i \mathrm{R}}-c_{i \mathrm{~L}}}{l} x\right) \frac{\mathrm{d} \psi}{\mathrm{d} x}
$$

Rearranging terms

$$
\mathrm{d} \psi=-\frac{\sum_{i} z_{i} D_{i} \frac{c_{i \mathrm{R}}-c_{i \mathrm{~L}}}{l}}{\sum_{i} z_{i}^{2} D_{i}\left(c_{i \mathrm{~L}}+\frac{c_{i \mathrm{R}}-c_{i \mathrm{~L}}}{l} x\right)} \mathrm{d} x
$$

The integration of the differencial equation gives the differential electrical potential at the liquid junction

$$
\psi_{\mathrm{R}}-\psi_{\mathrm{L}}=-\int_{0}^{l} \frac{\sum_{i} z_{i} D_{i} \frac{c_{i \mathrm{R}}-c_{i \mathrm{~L}}}{l}}{\sum_{i} z_{i}^{2} D_{i}\left(c_{i \mathrm{~L}}+\frac{c_{i \mathrm{R}}-c_{i \mathrm{~L}}}{l} x\right)} \mathrm{d} x
$$


To perform this integration we consider first the derivative of the function

$$
f(x)=\ln \left[\sum_{i} z_{i}^{2} D_{i}\left(c_{i \mathrm{~L}}+\frac{c_{i \mathrm{R}}-c_{i \mathrm{~L}}}{l} x\right)\right]
$$

which is

$$
\frac{\mathrm{d} f}{\mathrm{~d} x}=\frac{\sum_{i} z_{i}^{2} D_{i} \frac{c_{i \mathrm{R}}-c_{i \mathrm{~L}}}{l}}{\sum_{i} z_{i}^{2} D_{i}\left(c_{i \mathrm{~L}}+\frac{c_{i \mathrm{R}}-c_{i \mathrm{~L}}}{l} x\right)}
$$

Observe that the numerator of $\mathrm{d} f / \mathrm{d} x$ is a constant that can be changed into the numerator of the integrand in equation (B.7). We just have to define the function

$$
g(x)=\frac{\sum_{i} z_{i} D_{i} \frac{c_{i \mathrm{R}}-c_{i \mathrm{~L}}}{l}}{\sum_{i} z_{i}^{2} D_{i} \frac{c_{i \mathrm{R}}-c_{i \mathrm{~L}}}{l}} f(x)
$$

whose derivative

$$
\frac{\mathrm{d} g}{\mathrm{~d} x}=\frac{\sum_{i} z_{i} D_{i} \frac{{ }{ }_{i \mathrm{R}}-c_{i \mathrm{~L}}}{l}}{\sum_{i} z_{i}^{2} D_{i} \frac{c_{i \mathrm{R}}-c_{i \mathrm{E}}}{l}} \frac{\sum_{i} z_{i}^{2} D_{i} \frac{{ }{ }_{i \mathrm{R}}-c_{i \mathrm{E}}}{l}}{\sum_{i} z_{i}^{2} D_{i}\left(c_{i \mathrm{~L}}+\frac{c_{i \mathrm{R}}-c_{i \mathrm{~L}}}{l} x\right)}
$$

coincides with the integrand of equation (B.7). We have then

$$
\psi_{\mathrm{R}}-\psi_{\mathrm{L}}=-\frac{\sum_{i} z_{i} D_{i} \frac{c_{i \mathrm{R}}-c_{i \mathrm{~L}}}{l}}{\sum_{i} z_{i}^{2} D_{i} \frac{{ }^{c_{i \mathrm{R}}}-c_{i \mathrm{~L}}}{l}} \ln \left[\left.\sum_{i} z_{i}^{2} D_{i}\left(c_{i \mathrm{~L}}+\frac{c_{i \mathrm{R}}-c_{i \mathrm{~L}}}{l} x\right)\right|_{0} ^{l}\right]
$$

or

$$
\psi_{\mathrm{R}}-\psi_{\mathrm{L}}=-\frac{\sum_{i} z_{i} D_{i}\left(c_{i \mathrm{R}}-c_{i \mathrm{~L}}\right)}{\sum_{i} z_{i}^{2} D_{i}\left(c_{i \mathrm{R}}-c_{i \mathrm{~L}}\right)} \ln \left(\frac{\sum_{i} z_{i}^{2} D_{i} c_{i \mathrm{R}}}{\sum_{i} z_{i}^{2} D_{i} c_{i \mathrm{~L}}}\right)
$$

which in known as the Henderson equation. 